\title{
Prognostic value of 'late' electroencephalography recordings in patients with cardiopulmonal resuscitation after cardiac arrest
}

\author{
Jakob I. Doerrfuss ${ }^{1}$ D $\cdot$ Alexander B. Kowski ${ }^{1} \cdot$ Martin Holtkamp ${ }^{1} \cdot$ Moritz Thinius $^{1}$. Christoph Leithner ${ }^{1}$. \\ Christian Storm ${ }^{2}$
}

Received: 20 February 2021 / Revised: 2 April 2021 / Accepted: 5 April 2021 / Published online: 19 April 2021

(c) The Author(s) 2021

\begin{abstract}
Background Electroencephalography (EEG) significantly contributes to the neuroprognostication after resuscitation from cardiac arrest. Recent studies suggest that the prognostic value of EEG is highest for continuous recording within the first days after cardiac arrest. Early continuous EEG, however, is not available in all hospitals. In this observational study, we sought to evaluate the predictive value of a 'late' EEG recording 5-14 days after cardiac arrest without sedatives.

Methods We retrospectively analyzed EEG data in consecutive adult patients treated at the medical intensive care units (ICU) of the Charité-Universitätsmedizin Berlin. Outcome was assessed as cerebral performance category (CPC) at discharge from ICU, with an unfavorable outcome being defined as CPC 4 and 5.

Results In 187 patients, a 'late' EEG recording was performed. Of these patients, 127 were without continuous administration of sedative agents for at least $24 \mathrm{~h}$ before the EEG recording. In this patient group, a continuously suppressed background activity $<10 \mu \mathrm{V}$ predicted an unfavorable outcome with a sensitivity of $31 \%$ (95\% confidence interval (CI) 20-45) and a specificity of 99\% (95\% CI 91-100). In patients with suppressed background activity and generalized periodic discharges, sensitivity was $15 \%$ (95\% CI 7-27) and specificity was 100\% (95\% CI 94-100). GPDs on unsuppressed background activity were associated with a sensitivity of $42 \%$ (95\% CI 29-46) and a specificity of $92 \%$ (95\% CI 82-97).

Conclusions A 'late' EEG performed 5 to 14 days after resuscitation from cardiac arrest can aide in prognosticating functional outcome. A suppressed EEG background activity in this time period indicates poor outcome.
\end{abstract}

Keywords EEG $\cdot$ Predictive value of tests $\cdot$ Resuscitation $\cdot$ Outcome $\cdot$ Targeted temperature management

\section{Introduction}

Predicting neurological outcome after cardiopulmonal resuscitation (CPR) following cardiac arrest (CA) is important for determining further treatment choices and decisions regarding withdrawal of life-sustaining therapy. Diagnostic workup after CA to assess prognosis consists of clinical examination, short-latency somatosensory evoked potentials

JakobI. Doerrfuss and AlexanderB. Kowski have contributed equally.

Jakob I. Doerrfuss

jakob.doerrfuss@charite.de

1 Department of Neurology and Experimental Neurology, Charité-Universitätsmedizin Berlin, Berlin, Germany

2 Department of Nephrology and Medical Intensive Care, Charité-Universitätsmedizin Berlin, Berlin, Germany
(SSEPs), measurement of neuron-specific enolase (NSE), brain imaging and electroencephalography (EEG). This outcome prediction is especially important in patients who remain comatose after resuscitation from CA [1].

Several EEG parameters are associated with a poor prognosis after CA [2]. Recent studies have suggested that 'early' continuous EEG can detect poor outcome with higher sensitivity than 'late' EEG $[3,4]$. In early EEG measurements, the sensitivity for the prediction of unfavorable outcome decreased after $>12 \mathrm{~h}$ following resuscitation from CA while the specificity remained robust [4]. However, EEG measurements are not broadly available at all times. EEG activity underlies a natural evolution following CA and could also be altered due to effects of sedative medication $[5,6]$. This has become of particular importance since the routine implementation of targeted temperature management (TTM). According to current guidelines, almost all survivors after CA not responding to painful stimuli should receive 
TTM in a range of $32-36^{\circ} \mathrm{C}$ for at least $24 \mathrm{~h}$, which requires administration of sedatives [1].

Current guidelines provide different recommendations regarding the timing of EEG recordings for the assessment of prognosis after CA: an advisory statement from the European Resuscitation Council from 2015 recommends measuring EEG $72 \mathrm{~h}$ after recovery of spontaneous circulation (ROSC) [7]. In contrast, the task force for Belgian recommendations suggests measuring EEG 'as soon as possible' [8].

In this study, we seek to determine the clinical significance of a 'late' EEG recording between days 5 and 14 after resuscitation from $\mathrm{CA}$ in comatose patients. We hypothesize that the evaluation of such a 'late' EEG can aide in predicting functional outcome and that the predictive value of 'late' EEG recordings is higher in patients not under continuous sedative medication.

\section{Methods}

\section{Data source and patients}

We analyzed data from consecutive adult patients (aged 18 or above) admitted to the medical intensive care units (ICU) of the Charité-Universitätsmedizin Berlin (at Campus Virchow-Klinikum) after successful resuscitation from CA between June 1st 2009 and May 31st 2013. At that time, EEG recordings performed between days 5 and 14 after successful resuscitation from CA were part of clinical routine. Late EEG recordings were requested by ICU physicians only in patients still comatose. Institutional approval for this retrospective, observational study was provided by the local Ethics committee (EA2/115/13).

Clinical data were derived from a large prospective database described previously [9]. These data comprised age at CA, sex, location of CPR (out-of-hospital vs. in-hospital), initial cardiac rhythm (shockable vs. non-shockable), time to ROSC (tROSC), NSE at day 3 after CA, SSEP data ( $24 \mathrm{~h}$ to 4 days post CA), EEG data (5 to 14 days post CA), and $\mathrm{CPC}$ score at discharge. CPC scores were determined at the time of ICU discharge and prospectively documented in the database. Electroencephalography and SSEP data were assessed following strict criteria by three of the authors (JD, AK, MT) and, in uncertain cases, reassessed by two other authors (MH, CL).

\section{Primary and secondary outcomes}

Our primary study outcome parameter was cerebral performance category (CPC) at discharge from the ICU. We dichotomized CPC, defining a CPC score of 1-3 as favorable outcome and a CPC of 4 and 5 as unfavorable neurological outcome. This was done to avoid falsely allocating patients with prolonged recovery to the unfavorable outcome group. To improve comparability to other studies, we performed a supplementary analysis where a CPC score of 3 (severe cerebral disability) at discharge was considered an unfavorable outcome.

\section{Definition of parameters}

\section{EEG}

5 to 14 days after CA, a 'late' EEG with video-monitoring was performed for $20 \mathrm{~min}$ using a digital 21-channel recording system (Nihon Kohden, Japan) with the international 10-20 system for electrode placement and reviewed with standard montages. EEG data were analyzed for four predefined features that were previously described to be associated with unfavorable outcome after CPR [10]:

(1) Suppressed background activity without discharges was defined as amplitude $<10 \mu \mathrm{V}$, during the entire recording, resulting in non-assessable frequency, without disruption by focal or generalized discharges.

(2) Generalized periodic discharges (GPDs) on suppressed background activity was defined as amplitude of background activity $<10 \mu \mathrm{V}$ during the entire recording which was interrupted by GPDs defined according to the American Clinical Neurophysiology Society's (ACNS) Standardized Critical Care EEG Terminology. GPDs are characterized by periodically recurring monomorphic discharges with waveforms of a duration $<0.5 \mathrm{~s}$ regardless of number of phases or waveforms lasting $\geq 0.5 \mathrm{~s}$ with a maximum of three phases [11]. An example of GPDs on suppressed background activity is given in Fig. 1.

(3) GPDs on unsuppressed background activity was defined as amplitude of background activity $\geq 10 \mu \mathrm{V}$ which was interrupted by GPDs.

(4) Burst-suppression pattern (BSP) was defined in accordance to the ACNS standardized critical care EEG terminology as generalized periodic, recurring high-voltage discharges occurring with more than three phases and a duration of $\geq 0.5 \mathrm{~s}$ against a suppressed background activity [11].

SSEPs Median nerve somatosensory evoked potentials (SSEPs) were recorded using Nihon Kohden Neuropack four mini system (Nihon Kohden, Japan). Stimulation intensity was adjusted to produce a peripheral response (thumb twitch). For each recording $>500$ SSEPs were performed and averaged. The highest amplitude of a reproducible cortical potential ( $>4.5 \mathrm{~ms}$ after the N13 peak) was measured (peak to peak or baseline to peak). N20 was only deter- 


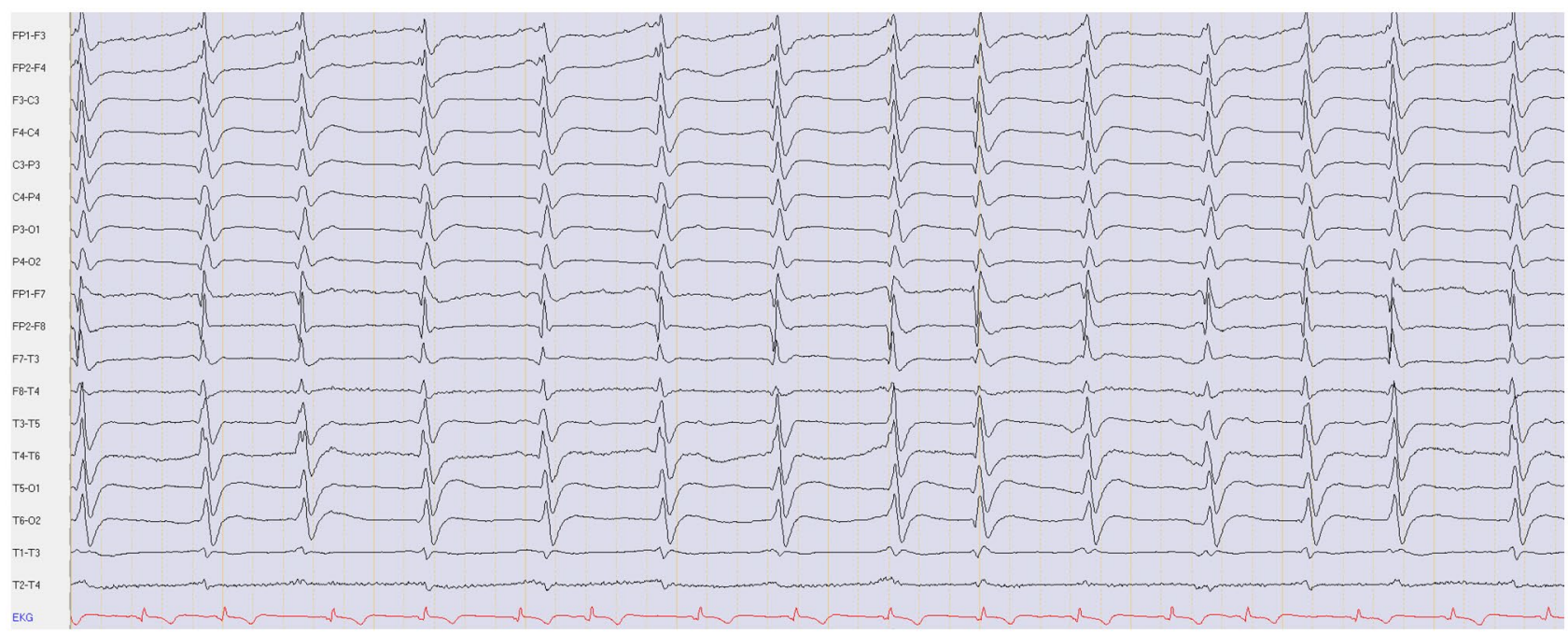

Fig. 1 Example of GPDs on suppressed background activity

mined as bilaterally absent, if cortical noise level was below $0.25 \mu \mathrm{V}$ and there were no reproducible cortical potentials but reproducible spinal and peripheral potentials. SSEPs were recorded $24 \mathrm{~h}$ to 4 days after resuscitation from CA.

\section{NSE}

Serum concentration of NSE was determined at day 3 after CA. A threshold of $90 \mu \mathrm{g} / \mathrm{l}$ was applied for calculation of sensitivities and specificities [12].

\section{Standardized care at the intensive care units}

All patients were treated with TTM following a strict inhouse protocol along the lines of the International Liaison Committee on Resuscitation recommendations [13]. The target temperature of $33{ }^{\circ} \mathrm{C}$ was maintained for $24 \mathrm{~h}$ followed by a controlled re-warming rate of $0.25^{\circ} \mathrm{C}$ per hour. Fever had to be avoided for further $72 \mathrm{~h}$. In all patients, a computer controlled surface cooling device (Arctic Sun ${ }^{\mathrm{TM}}$ Temperature Management System, C.R.BARD, Colorado, USA) was used. A combination of intravenous midazolam and fentanyl or isoflurane (volatile) and remifentanil was used for analgosedation.

\section{Withdrawal of life-sustaining therapy}

Withdrawal of life-sustaining therapy was always based on careful consideration of multimodal neurological prognostication and a considerable observation period. In 2011, an internal protocol for prognostication was established [14] and modified according to new evidence over time. The protocol is largely in line with the 2014 guidelines of the European Society of Intensive Care Medicine [7] and emphasizes multimodal prognostication and an observation period of at least 7 days in most cases. Patients' advanced directives and preferences communicated by relatives were taken into account. In a relevant subset of patients, withdrawal of life-sustaining therapy was not performed despite poor prognostic findings. These patients were discharged in coma or unresponsive wakefulness syndrome. We followed these patients in long-term and found no case of an unexpected late recovery, indicating a low probability of a self-fulfilling prophecy for our prognostication algorithm [15].

\section{Statistical analysis}

Categorical variables were analyzed with Pearson's $\chi^{2}$ or Fischer's test. Continuous data were checked for normal distribution using Kolmogorov-Smirnov test and were then presented as mean \pm standard deviation or median and interquartile range (IQR) where appropriate; Mann-Whitney $U$ test was used for continuous variables.

Sensitivity and specificity were calculated with $2 \times 2$ contingency tables and are presented with $95 \%$ confidence intervals (CI). For the identification of independent predictors for unfavorable outcome at ICU discharge, a binary logistic regression analysis (inclusion method: stepwise backward, $p<0.1$ [ $p$ in],$p<0.05$ [ $p$ out], iteration 20 , cutoff set 0.26 and constant was included) was used with clinically relevant covariates to estimate odds ratios (OR) with $95 \%$ CI. Parameters with quasi-complete separation were excluded from the logistic regression analysis.

Statistical analyses were performed with SPSS version 25 (IBM, Chicago, IL, USA). 


\section{Results}

\section{Patient population}

During the 4-year study period, 320 patients were treated with TTM after out-of-hospital or in-hospital CA and successful resuscitation. One hundred and thirty-three patients were excluded from the analysis because they were discharged or had died before the EEG was performed, because no EEG was recorded in the time window from day 5-14 after CA or because EEG recordings could not be retrieved (Fig. 2).

Our final analysis included 187 patients, mean age was $62 \pm 16$ years, $28 \%$ female, $71 \%$ with out-of-hospital CPR. Median CPC at discharge was 2 (interquartile range (IQR) $1-5), 41 \%$ of patients had an unfavorable outcome at discharge, and mortality rate was $27 \%$. Median time point of EEG recording was 8 days after CA (IQR 7-10), and median ICU length of stay was 21 days (IQR 13-33).

Comparing included and excluded patients, we found no significant differences concerning sex, age, and location of CPR. However, the initial presence of non-shockable rhythm, time to ROSC, mean NSE, CPC, and mortality showed significant group-differences (Table-e1, online only).

Of all 187 included patients, 127 were without continuous administration of sedative agents for at least $24 \mathrm{~h}$ before the EEG recording. In 60 patients, the EEG recording was performed under or within $24 \mathrm{~h}$ of continuous administration of sedatives. There were no statistically significant differences between both groups with regards to sex, age, proportion of

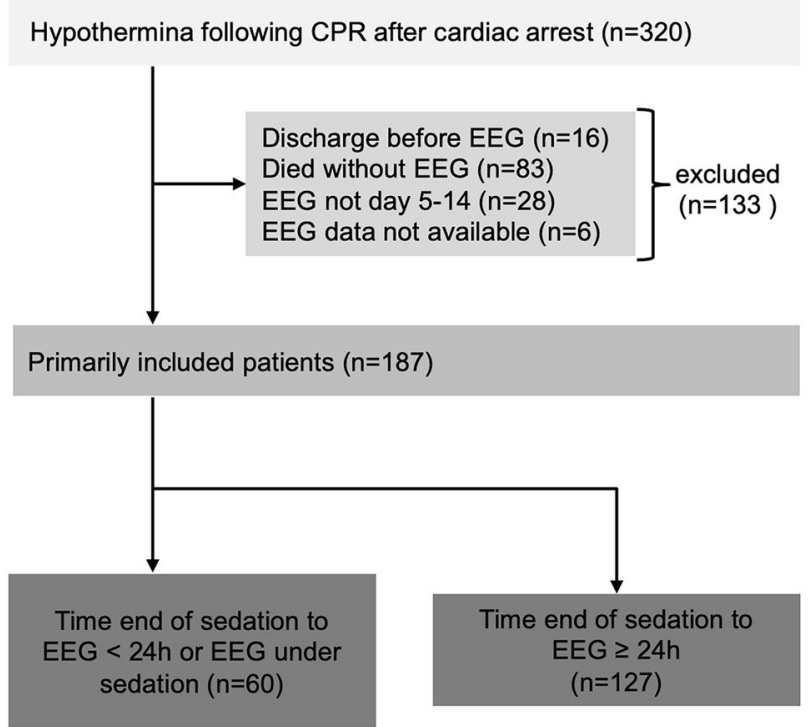

Fig. 2 Patient recruitment out-of-hospital CPR and initial shockable rhythm, NSE at day 3 , and clinical outcome (Table e-2, online only).

\section{Outcome prediction in patients without continuous administration of sedatives for $>24 \mathrm{~h}$ before EEG measurements}

In the 127 patients without continuous administration of sedatives, 55 patients (43\%) had an unfavorable outcome.

Seventeen patients with unfavorable outcome (31\%) had a continuously suppressed background activity with no discharges. A suppressed background activity with no discharges predicted unfavorable outcome with a sensitivity of $31 \%$ (95\% confidence interval [CI] 20-45) and a specificity of 99\% (95\% CI 91-100). GPDs on unsuppressed background activity were associated with a sensitivity of $42 \%$ (95\% CI 29-56) and a specificity of $92 \%$ (95\% CI 82-97) for the prediction of unfavorable outcome.

One patient with suppressed background activity without discharges and six patients with GPDs on unsuppressed background had a favorable outcome. Two of the patients with GPDs on unsuppressed background and favorable outcome had received a bolus of sedatives in the $24 \mathrm{~h}$ preceding the EEG recordings. The patient with suppressed background activity without discharges and favorable outcome was a 67 old female who was resuscitated from CA following pulmonal artery embolism. Sedation with midazolam and fentanyl was discontinued 4.5 days before the EEG recording and no bolus of sedative medication was given in the $48 \mathrm{~h}$ preceding the EEG recording. We provide a more detailed description of this case in the supplementary material.

No false positives were present in the eight patients who showed both GPDs and suppressed background activity (GPDs on suppressed background; sensitivity for the prediction of unfavorable outcome 15\% (95\% CI 7-27); specificity $100 \%$ (95\% CI 94-100). The prognostic parameters are summarized in Table 1; the analyses for an unfavorable outcome being defined as CPC $3-5$ are provided in the supplementary material (Table e3, online only).

The presence of suppressed background activity without discharges (OR 36.9, 95\% CI 3.3-414.3) and of GPDs on unsuppressed background (OR 57.3, 95\% CI 8.4-390.8) both independently predicted unfavorable outcome after CA, see Table 2 (for CPC 3-5 as unfavorable outcome see Table e4, online only).

In the patient group without continuous administration of sedatives for at least $24 \mathrm{~h}$ before the EEG measurement, 24 patients (19\%) had NSE values $>90 \mu \mathrm{g} / \mathrm{l} 3$ days after resuscitation from CA. The sensitivity for the prediction of an unfavorable outcome in patients with NSE $>90 \mu \mathrm{g} / \mathrm{l}$ was $42 \%$ (95\% CI 29-56) and specificity was $98 \%$ (95\% 
Table 1 Predictive values for unfavorable outcome (CPC 4 and 5)

\begin{tabular}{|c|c|c|c|c|c|c|c|}
\hline & $\begin{array}{l}\text { Fre- } \\
\text { quency }(n) \\
(n=127)\end{array}$ & Sensitivity (95\% CI) & Specificity (95\% CI) & True positive & False positive & True negative & False negative \\
\hline \multicolumn{8}{|c|}{ Patients without continuous sedative-administration for at least $24 \mathrm{~h}$} \\
\hline \multicolumn{8}{|l|}{ EEG parameters } \\
\hline $\begin{array}{l}\text { Suppressed back- } \\
\text { ground without } \\
\text { discharges }\end{array}$ & $14 \%(18)$ & $31 \%(20-45)$ & $99 \%(91-100)$ & 17 & 1 & 71 & 38 \\
\hline $\begin{array}{l}\text { GPDs on suppressed } \\
\text { background }\end{array}$ & $6 \%(8)$ & $15 \%(7-27)$ & $100 \%(94-100)$ & 8 & 0 & 72 & 47 \\
\hline $\begin{array}{l}\text { GPDs on unsup- } \\
\text { pressed background }\end{array}$ & $23 \%(29)$ & $42 \%(29-56)$ & $92 \%(82-97)$ & 23 & 6 & 66 & 32 \\
\hline \multicolumn{8}{|l|}{ Other parameters } \\
\hline $\mathrm{NSE}>90 \mu \mathrm{g} / 1$ & $19 \%(24)$ & $42 \%(29-56)$ & $98 \%(91-100)$ & 23 & 1 & 71 & 32 \\
\hline Bilateral absent SSEPs & $14 \%(18)$ & $33 \%(21-47)$ & $100 \%(94-100)$ & 18 & 0 & 72 & 37 \\
\hline \multicolumn{8}{|c|}{ Patients with continuous sedative-administration stopped less than 24 h before or with EEG during continuous sedation } \\
\hline \multicolumn{8}{|l|}{ EEG parameters } \\
\hline $\begin{array}{l}\text { Suppressed back- } \\
\text { ground without } \\
\text { discharges }\end{array}$ & $8 \%(5)$ & $23 \%(9-46)$ & $100 \%(89-100)$ & 5 & 0 & 38 & 17 \\
\hline $\begin{array}{l}\text { GPDs on suppressed } \\
\text { background }\end{array}$ & $5 \%(3)$ & $14 \%(4-36)$ & $100 \%(89-100)$ & 3 & 0 & 38 & 19 \\
\hline $\begin{array}{l}\text { GPDs on unsup- } \\
\text { pressed background }\end{array}$ & $20 \%(12)$ & $27 \%(12-50)$ & $84 \%$ (68-93) & 6 & 6 & 32 & 16 \\
\hline \multicolumn{8}{|l|}{ Other parameters } \\
\hline $\mathrm{NSE}>90 \mu \mathrm{g} / \mathrm{l}$ & $15 \%(9)$ & $32 \%(15-55)$ & $95 \%(81-99)$ & 7 & 2 & 36 & 15 \\
\hline Bilateral absent SSEPs & $7 \%(4)$ & $18 \%(6-41)$ & $100 \%(89-100)$ & 4 & 0 & 38 & 18 \\
\hline
\end{tabular}

$C P C$ cerebral performance category at intensive care unit discharge, $C I$ confidence interval, GPDs generalized periodic discharges, NSE neuronspecific enolase, SSEPs short-latency somatosensory evoked potentials

CI 91-100). One patient with NSE $>90 \mu \mathrm{g} / \mathrm{l}$ had a favorable outcome at discharge (CPC 1).

In the same patient group, SSEPs were bilateral absent in 18 patients $(14 \%)$. In no patient with favorable outcome, SSEPs were bilateral absent, resulting in a sensitivity for this outcome parameter of 33\% (95\% CI 21-47) and a specificity of $100 \%$ (95\% CI 94-100).

Late EEG correctly predicted unfavorable outcome in 23 patients in whom SSEPs were not bilaterally absent and NSE was $\leq 90 \mu \mathrm{g} / \mathrm{l}$ (4 patients with GPDs on unsuppressed background, 7 patients with suppressed background without discharges and 12 patients with GPDs on unsuppressed background). SSEPs were able to correctly predict unfavorable outcome in one patient with normal EEG and NSE $\leq 90 \mu \mathrm{g} / \mathrm{l}$. In all patients with unfavorable outcome and NSE $>90 \mu \mathrm{g} / \mathrm{l}$, either SSEPs or EEG parameters were also predictive of unfavorable outcome. In five patients with $\mathrm{CPC}>3$, no prognostic parameter was predictive of this unfavorable outcome.

\section{Outcome prediction in patients with continuous sedative-administration stopped $<24 \mathrm{~h}$ before or with EEG during continuous sedation}

Of the 60 patients with EEG recording within $24 \mathrm{~h}$ after administration of sedatives or with EEG recording during continuous sedation, 22 patients $(37 \%)$ had an unfavorable outcome. In this patient group, the sensitivity for the prediction of unfavorable outcome was lower for the EEG parameters 'suppressed background without discharges' and 'GPDs on unsuppressed background' parameters as compared to the group of patients with EEG without continuous administration of sedatives. The diagnostic accuracy of the EEG parameter 'GPDs on suppressed background' was not different between the two groups. Specificities of all outcome predictors were comparable between both patient groups (see Table 1). The analysis of predictors for CPC-based outcome is shown in Table 2. 
Table 2 Predictors for CPC-based outcome (CPC 1-3 vs 4 and 5)

\begin{tabular}{|c|c|c|c|c|}
\hline & \multicolumn{2}{|l|}{ CPC-based outcome } & \multirow[t]{3}{*}{ Univariate analyses } & \multirow{3}{*}{$\begin{array}{l}\text { Binary logistic regression, } \\
\operatorname{Exp}(\mathrm{B})[95 \% \mathrm{CI}]\end{array}$} \\
\hline & Favorable (CPC 1-3) & $\begin{array}{l}\text { Unfavorable } \\
\text { (CPC } 4 \text { and 5) }\end{array}$ & & \\
\hline & $n=72$ & $n=55$ & & \\
\hline \multicolumn{5}{|c|}{ Patients without continuous sedative-administration for at least $24 \mathrm{~h}$} \\
\hline \multicolumn{5}{|l|}{ Demographics and basic information: } \\
\hline Female sex, $n(\%)$ & $17(24)$ & $19(35)$ & $p=0.233^{\mathrm{a}}$ & Not included \\
\hline Age [years], median (IQR) & $63(52-73)$ & $70(61-75)$ & $p=0.052^{\mathrm{b}}$ & Not significant \\
\hline Out-of-hospital CPR, $n(\%)$ & $47(69)$ & $37(77)$ & $p=0.403$ & Not included \\
\hline Initial rhythm: not shockable, $n(\%)$ & $18(32)$ & $30(67)$ & $p=0.001^{\mathrm{a}}$ & $5.9[1.3-26.5]$ \\
\hline TROSC [min], median (IQR) & $12(7.5-20)$ & $18(10-28)$ & $p=0.055^{\mathrm{b}}$ & Not significant \\
\hline \multicolumn{5}{|l|}{ NSE day 3} \\
\hline$[\mu \mathrm{g} / \mathrm{l}]$, median $(\mathrm{IQR})$ & $20(15-27)$ & $68(33-130)$ & $p<0.001^{\mathrm{b}}$ & Not included \\
\hline $\mathrm{NSE}>90 \mu \mathrm{g} / \mathrm{l}, n(\%)$ & $1(1)$ & $23(42)$ & $p<0.001^{\mathrm{a}}$ & $56.3[4.5-704.7]$ \\
\hline \multicolumn{5}{|l|}{ Electrophysiology: } \\
\hline \multicolumn{5}{|l|}{ EEG } \\
\hline Suppressed background without discharges, $n(\%)$ & $1(1)$ & $17(31)$ & $p<0.001^{\mathrm{a}}$ & $36.9[3.3-414.3]$ \\
\hline GPDs on suppressed background, $n(\%)$ & $0(0)$ & $8(15)$ & $p<0.001^{\mathrm{a}}$ & Not included ${ }^{\dagger}$ \\
\hline GPDs on unsuppressed background, $n(\%)$ & $6(8)$ & $23(42)$ & $p<0.001^{\text {a }}$ & $\mathbf{5 7 . 3}[8.4-390.8]$ \\
\hline \multicolumn{5}{|l|}{ SSEP } \\
\hline \multirow[t]{2}{*}{ SSEPs bilateral absent, $n(\%)$} & $0(0)$ & $18(33)$ & $p<0.001^{\mathrm{c}}$ & Not included ${ }^{\dagger}$ \\
\hline & $n=38$ & $n=22$ & & \\
\hline
\end{tabular}

Patients with continuous sedative-administration stopped less than 24 h before or with EEG during continuous sedation

Demographics and basic information:

\begin{tabular}{|c|c|c|c|c|}
\hline Female sex, $n(\%)$ & $11(29)$ & $6(27)$ & $p=1.000^{\mathrm{a}}$ & Not included \\
\hline Age [years], median (IQR) & $61(51-70)$ & $61(55-73)$ & $p=0.634^{\mathrm{b}}$ & Not significant \\
\hline Out-of-hospital CPR, $n(\%)$ & $25(66)$ & $15(75)$ & $p=0.559^{\mathrm{a}}$ & Not included \\
\hline Initial rhythm: not shockable, $n(\%)$ & $10(31)$ & $9(53)$ & $p=0.218^{\mathrm{a}}$ & Not significant \\
\hline TROSC [min], median (IQR) & $12(10-20)$ & $19(12-24)$ & $p=0.118^{\mathrm{b}}$ & Not significant \\
\hline \multicolumn{5}{|l|}{ NSE day 3} \\
\hline$[\mu \mathrm{g} / \mathrm{l}]$, median $(\mathrm{IQR})$ & $21(13-28)$ & $44(26-111)$ & $p<0.001^{\mathrm{b}}$ & Not included \\
\hline $\mathrm{NSE}>90 \mu \mathrm{g} / \mathrm{l}, n(\%)$ & $2(5)$ & $7(32)$ & $p=0.009^{\mathrm{a}}$ & $6.6[1.1-39.1]$ \\
\hline \multicolumn{5}{|l|}{ Electrophysiology: } \\
\hline \multicolumn{5}{|l|}{ EEG } \\
\hline Suppressed background without discharges, $n(\%)$ & $0(0)$ & $5(23)$ & $p=0.005^{\mathrm{a}}$ & Not included ${ }^{\dagger}$ \\
\hline GPDs on suppressed background, $n(\%)$ & $0(0)$ & $3(14)$ & $p=0.045^{\mathrm{a}}$ & Not included ${ }^{\dagger}$ \\
\hline GPD on unsuppressed background, $n(\%)$ & $6(16)$ & $6(27)$ & $p=0.327^{\mathrm{a}}$ & Not significant \\
\hline \multicolumn{5}{|l|}{ SSEP } \\
\hline SSEPs bilateral absent, $n(\%)$ & $0(0)$ & $4(18)$ & $p=0.015^{\mathrm{a}}$ & Not included ${ }^{\dagger}$ \\
\hline
\end{tabular}

The $p$-values and odds-ratios of statistically significant parameters are written in bold

$C P C$ cerebral performance category at intensive care unit discharge, IQR interquartile range, $t R O S C$ time to resuscitate spontaneous circulation, $N S E$ neuron-specific enolase, $C P R$ cardiopulmonal resuscitation

${ }^{\text {a}}$ Fisher's exact (two-sided)

${ }^{\mathrm{b}}$ Mann-Whitney $U$ test (two-sided)

${ }^{\mathrm{c}}$ Pearson's $\chi^{2}$ test (two-sided). $\operatorname{Exp}(B)$ odds ratio; $C I$ confidence interval

${ }^{\dagger}$ The variables were not included to avoid quasi-complete separation in the model 
Only three patients had a burst-suppression-pattern at the time of EEG recording. Therefore, we omitted this EEG pattern from our statistical analysis.

\section{Outcome prediction with unfavorable outcome defined as CPC 3-5}

When an unfavorable outcome was more broadly defined as CPC 3-5, five patients shifted to the unfavorable outcome group (four patients without continuous administration of sedatives for at least $24 \mathrm{~h}$ before the EEG measurement, one patient with sedative administration). With this alternative definition, 59 patients (46\%) without continuous sedativeadministration had an unfavorable outcome. In this patient group, suppressed background without discharges predicted unfavorable outcome with a sensitivity of $29 \%$ (95\% CI $18-42$ ) and a specificity of $99 \%$ (95\% CI 91-100). GPDs on suppressed background had a sensitivity of $14 \%$ (95\% CI 6-26) and a specificity of $100 \%$ (95\% CI 93-100). The sensitivity for GPDs on unsuppressed background was $41 \%$ (95\% CI 28-54) and the specificity was $93 \%$ (95\% CI 83-97).

All results with this alternative outcome definition including predictive values for all parameters and analysis of predictors of CPC-based outcome are listed in the supplementary material (Tables e3 and e4).

\section{Discussion}

In this study, a suppressed background activity without discharges in a 'late' EEG recording 5-14 days after CPR following CA had a specificity of $99 \%$ for the prediction of unfavorable outcome in patients without continuous administration of sedative medication for at least $24 \mathrm{~h}$ prior to the EEG recording. When both GPDs and a suppressed background activity (GPDs on suppressed background) were present, there were no false-positives, although the number of patients in this group was small $(n=9)$, resulting in a broader confidence interval. The predictive values of these EEG parameters were comparable to bilateral absent SSEPs $24 \mathrm{~h}$ to 4 days post CA or to a NSE $>90 \mu \mathrm{g} / \mathrm{l} 3$ days after CA.

With a specificity of $92 \%$ and a false-positive rate of $8 \%$, the predictive value of GPDs on unsuppressed background activity does not seem suitable as a parameter for the detection of an unfavorable outcome after CPR.

The sensitivities of the EEG parameters 'suppressed background activity without discharges' and 'GPDs on unsuppressed background activity' were lower in patients in whom continuous administration of sedatives was stopped less than $24 \mathrm{~h}$ before the EEG recording while GPDs on unsuppressed background did not seem to be affected by sedative medication. While the sensitivities of most outcome predictors were reduced in patients under influence of sedative medication, the specificity of these outcome predictors remained mostly similar.

EEG plays an important role in prognostication after CA, and its importance has not declined since the introduction of TTM into clinical routine [10, 16, 17]. The diagnostic yield of EEG recordings in patients with TTM is time-dependent [4-6]. Focusing on a 'late' EEG recording, presumably associated with a lower load of sedative drugs, our study extends prior reports on time specificity of EEG patterns to 5-14 days after CA.

One patient with suppressed EEG background activity without discharges, who was not sedated during or $24 \mathrm{~h}$ before the EEG measurement, had a favorable functional outcome at discharge. This result is surprising as in other studies no patient with EEG background activity $<10 \mu \mathrm{V}$ had a favorable functional outcome $[3,18]$. In these studies, EEG was performed within $24 \mathrm{~h}$ after cardiac arrest. While low voltage EEG $<20 \mu \mathrm{V}$ is present in up to $10 \%$ of the general population and is often considered a normal variant, suppressed EEG background activity $(<10 \mu \mathrm{V})$ is regarded as a malignant EEG feature unequivocally associated with unfavorable outcome $[19,20]$. We provide a more detailed description of the patient in the supplementary material. Summing up, the clinical course of the patient provides no clear explanation for the suppressed background activity. However, we cannot entirely exclude that the patient was administered a bolus of sedatives in the hours prior to EEG recording which was not documented in the electronic chart.

Suppressed background activity without discharges and the presence of GPDs on unsuppressed background both independently predicted unfavorable outcome. The presence of one of these parameters correctly predicted unfavorable outcome in 11 patients in whom SSEPs and NSE were unpredictive of unfavorable outcome. The pathophysiology underlying GPDs is not yet fully understood. GPDs are considered to be a consequence of loss of inhibitory interneurons, which are in particular vulnerable to hypoxia $[21,22]$. The predictive value of GPDs in patients with hypoxic ischemic brain damage has been described previously in a group of 119 patients after CA in whom the EEG was performed within the first 14 days (mean 3.8 days) after the event [23]. In that study, a similar rate of GPDs after CA was detected.

In comparison to other studies in which an EEG was performed early after CA, there was a somewhat higher rate of suppressed background activity with GPDs [10,17] and suppressed background activity without discharges [17] in our patients with unfavorable outcome. In contrast to studies with EEG examinations performed early after CA [10, 17], burst suppression patterns were only rarely detected in our study $(n=3)$. Our findings could hint towards an increased relevance of GPDs and a suppressed background activity in late EEG recordings, when these EEG patterns are not 
modified by sedative drugs which are required during TTM. This hypothesis corresponds with our finding of a diminished sensitivity for both EEG parameters in patients under sedative medication. These results are also in line with a recently published study focusing on EEG reactivity, the significance of this parameter for the prediction of unfavorable outcome after CA was reduced in patients under sedative medication [24]. The reduced sensitivity of EEG parameters in patients under sedative medication could also be explained alternatively: patients with reduced brain activity could be in less 'need' of sedative medication while patients with more brain activity could require more sedative medication. Thus, sedation would not be the cause of a reduced sensitivity for the detection of unfavorable outcome but it would be a sign of a certain amount of brain activity which might be associated with unfavorable outcome but might be high enough to prevent suppressed background activity.

BSPs, especially those with 'identical bursts', recorded in the first $72 \mathrm{~h}$ after CA are an important predictor for unfavorable outcome [6]. However, it was demonstrated that these BSPs evolve into less specific EEG patterns early after CA [25], which may explain their low frequency in our patient population.

There are limitations to consider. First, a comparison of the results of an 'early' vs. a 'late' EEG recording would have been desirable. Second, we only have data on outcome at ICU discharge and no data on long-term outcome. To avoid assigning patients with prolonged recovery falsely to the 'unfavorable outcome group', we considered a CPC of 3 to be a favorable outcome. There were no relevant changes to our results when the assignment of a CPC of 3 was changed to the unfavorable outcome group. Third, although we accounted for sedative drugs, we did not assess intake of classical antiseizure medication, which about $9 \%$ of ICU patients receive [26] and which could also have influenced EEG findings [27]. Fourth, we cannot exclude that in patients in whom sedatives had been withdrawn $\geq 24 \mathrm{~h}$ before the EEG recording, there was still some effect of these lipophilic substances after prolonged release and redistribution from fatty tissue. What is more, in 28 subjects (22\%) in the patient group without continuous administration of sedatives, a bolus administration of either benzodiazepines or propofol was documented, which could also have influenced EEG results. Fifth, assessing outcome late after CA in a clinical study bears the risk of selection bias. At 5 days after CA, a considerable proportion of patients with severe hypoxic encephalopathy have already died. Likewise, it is possible that patients with mild hypoxic encephalopathy already have been discharged from the ICU at this time point. Therefore, we cannot rule out that our study includes a relatively low proportion of patients with either severe or very mild hypoxic encephalopathy. Thus, our patient selection most likely differs from those of studies on early EEG after CA. This could be an explanation for the relatively high proportion of patients with suppressed EEG background activity. Lastly, a major possible confounding factor in most studies assessing prognostication after CA is that of self-fulfilling prophecy as the results of the EEG examinations were known to the caregivers and, therefore, are likely to have influenced decisions regarding continuation or withdrawal of further therapy [28].

In summary, suppressed background activity without discharges and suppressed background activity with GPDs in a 'late' EEG recording 5-14 days after resuscitation from CA predict unfavorable outcome in patients not under continuous administration of sedatives. A relevant minority of patients with GPDs on unsuppressed background activity may regain consciousness within the next days.

Supplementary Information The online version contains supplementary material available at https://doi.org/10.1007/s00415-021-10549-y.

Author contributions Conceptualization: JID, ABK, MH, and CS. Formal analysis: JID and ABK. Investigation: JID, ABK, MT, MH, and CL. Resources: ABK and CS. Visualization: JID and ABK. Writing — original draft: JID and ABK. Writing—review and editing: MT, MH, CL, and CS. Supervision: MH and CS. JID and ABK contributed equally to this work. All authors approved the final manuscript.

Funding Open Access funding enabled and organized by Projekt DEAL. This study was not supported by external funding.

Availability of data and materials The data that support the findings of this study are available on request from the corresponding author. The data are not publicly available due to privacy or ethical restrictions.

\section{Declarations}

Conflict of interest JID, ABK, and MT declared no conflicts of interest with respect to the research, authorship, and/or publication of this article; MH reports personal fees from Arvelle, Bial, Desitin, Eisai, GW Pharma, UCB, and Zogenix, outside the submitted work; CL reports institutional fees from Bard Medical, Pfizer, and Zoll, personal fees from Edwards Lifesciences, outside the submitted work; CS reports personal fees from BD Bard, Braincool, Pfizer, Xenios, and Sedana Medical, outside the submitted work.

Ethical approval Institutional approval for this observational study was provided by the local Ethics committee (EA2/115/13) of Charité-Universitätsmedizin Berlin.

Open Access This article is licensed under a Creative Commons Attribution 4.0 International License, which permits use, sharing, adaptation, distribution and reproduction in any medium or format, as long as you give appropriate credit to the original author(s) and the source, provide a link to the Creative Commons licence, and indicate if changes were made. The images or other third party material in this article are included in the article's Creative Commons licence, unless indicated otherwise in a credit line to the material. If material is not included in the article's Creative Commons licence and your intended use is not permitted by statutory regulation or exceeds the permitted use, you will need to obtain permission directly from the copyright holder. To view a copy of this licence, visit http://creativecommons.org/licenses/by/4.0/. 


\section{References}

1. Nolan JP, Soar J, Cariou A, Cronberg T, Moulaert VR, Deakin CD, Bottiger BW, Friberg H, Sunde K, Sandroni C, European Resuscitation C, European Society of Intensive Care M (2015) European resuscitation council and european society of intensive care medicine 2015 guidelines for post-resuscitation care. Intensive Care Med 41(12):2039-2056. https://doi.org/10.1007/ s00134-015-4051-3

2. Muhlhofer W, Szaflarski JP (2018) Prognostic value of EEG in patients after cardiac arrest-an updated review. Curr Neurol Neurosci Rep 18(4):16. https://doi.org/10.1007/s11910-018-0826-6

3. Hofmeijer J, Beernink TM, Bosch FH, Beishuizen A, TjepkemaCloostermans MC, van Putten MJ (2015) Early EEG contributes to multimodal outcome prediction of postanoxic coma. Neurology 85(2):137-143. https://doi.org/10.1212/WNL.0000000000001742

4. Ruijter BJ, Tjepkema-Cloostermans MC, Tromp SC, van den Bergh WM, Foudraine NA, Kornips FHM, Drost G, Scholten E, Bosch FH, Beishuizen A, van Putten M, Hofmeijer J (2019) Early electroencephalography for outcome prediction of postanoxic coma: a prospective cohort study. Ann Neurol 86(2):203-214. https://doi. org/10.1002/ana.25518

5. Carrai R, Grippo A, Scarpino M, Spalletti M, Cossu C, Lanzo G, Peris A, Cianchi G, Batacchi S, Valente S, Gensini G, Amantini A (2016) Time-dependent and independent neurophysiological indicators of prognosis in post-anoxic coma subjects treated by therapeutic hypothermia. Minerva Anestesiol 82(9):940-949

6. Spalletti M, Carrai R, Scarpino M, Cossu C, Ammannati A, Ciapetti M, Tadini Buoninsegni L, Peris A, Valente S, Grippo A, Amantini A (2016) Single electroencephalographic patterns as specific and time-dependent indicators of good and poor outcome after cardiac arrest. Clin Neurophysiol 127(7):2610-2617. https://doi.org/10. 1016/j.clinph.2016.04.008

7. Sandroni C, Cariou A, Cavallaro F, Cronberg T, Friberg H, Hoedemaekers C, Horn J, Nolan JP, Rossetti AO, Soar J (2014) Prognostication in comatose survivors of cardiac arrest: an advisory statement from the European Resuscitation Council and the European Society of Intensive Care Medicine. Resuscitation 85(12):1779-1789. https://doi.org/10.1016/j.resuscitation.2014.08.011

8. Taccone FS, Baar I, De Deyne C, Druwe P, Legros B, Meyfroidt G, Ossemann M, Gaspard N (2017) Neuroprognostication after adult cardiac arrest treated with targeted temperature management: task force for Belgian recommendations. Acta Neurol Belg 117(1):3-15. https://doi.org/10.1007/s13760-017-0755-1

9. Endisch C, Storm C, Ploner CJ, Leithner C (2015) Amplitudes of SSEP and outcome in cardiac arrest survivors: a prospective cohort study. Neurology 85(20):1752-1760. https://doi.org/10.1212/WNL. 0000000000002123

10. Westhall E, Rossetti AO, van Rootselaar AF, Wesenberg Kjaer T, Horn J, Ullen S, Friberg H, Nielsen N, Rosen I, Aneman A, Erlinge D, Gasche Y, Hassager C, Hovdenes J, Kjaergaard J, Kuiper M, Pellis T, Stammet P, Wanscher M, Wetterslev J, Wise MP, Cronberg T, investigators TT-t (2016) Standardized EEG interpretation accurately predicts prognosis after cardiac arrest. Neurology 86(16):1482-1490. https://doi.org/10.1212/WNL.0000000000 002462

11. Hirsch LJ, Fong MWK, Leitinger M, LaRoche SM, Beniczky S, Abend NS, Lee JW, Wusthoff CJ, Hahn CD, Westover MB, Gerard EE, Herman ST, Haider HA, Osman G, Rodriguez-Ruiz A, Maciel CB, Gilmore EJ, Fernandez A, Rosenthal ES, Claassen J, Husain AM, Yoo JY, So EL, Kaplan PW, Nuwer MR, van Putten M, Sutter R, Drislane FW, Trinka E, Gaspard N (2021) American clinical neurophysiology society's standardized critical care EEG terminology: 2021 version. J Clin Neurophysiol 38(1):1-29. https://doi.org/ 10.1097/WNP.0000000000000806
12. Streitberger KJ, Leithner C, Wattenberg M, Tonner PH, Hasslacher J, Joannidis M, Pellis T, Di Luca E, Fodisch M, Krannich A, Ploner CJ, Storm C (2017) Neuron-specific enolase predicts poor outcome after cardiac arrest and targeted temperature management: a multicenter study on 1,053 patients. Crit Care Med 45(7):1145-1151. https://doi.org/10.1097/CCM.0000000000002335

13. Nolan JP, Morley PT, Vanden Hoek TL, Hickey RW, Kloeck WG, Billi J, Bottiger BW, Morley PT, Nolan JP, Okada K, Reyes C, Shuster M, Steen PA, Weil MH, Wenzel V, Hickey RW, Carli P, Vanden Hoek TL, Atkins D, International Liaison Committee on R (2003) Therapeutic hypothermia after cardiac arrest: an advisory statement by the advanced life support task force of the International Liaison Committee on Resuscitation. Circulation 108(1):118-121. https:// doi.org/10.1161/01.CIR.0000079019.02601.90

14. Leithner C, Storm C, Hasper D, Ploner CJ (2012) Prognose der Hirnfunktion nach kardiopulmonaler Reanimation und therapeutischer Hypothermie (Prognosis for Brain Function Following Cardiopulmonary Resuscitation and Therapeutic Hypothermia). Aktuelle Neurologie 39(03):145-154

15. Petzinka VN, Endisch C, Streitberger KJ, Salih F, Ploner CJ, Storm C, Nee J, Leithner C (2018) Unresponsive wakefulness or coma after cardiac arrest-a long-term follow-up study. Resuscitation 131:121-127. https://doi.org/10.1016/j.resuscitation.2018.07.007

16. Rossetti AO, Carrera E, Oddo M (2012) Early EEG correlates of neuronal injury after brain anoxia. Neurology 78(11):796-802. https://doi.org/10.1212/WNL.0b013e318249f6bb

17. Backman S, Cronberg T, Friberg H, Ullen S, Horn J, Kjaergaard J, Hassager C, Wanscher M, Nielsen N, Westhall E (2018) Highly malignant routine EEG predicts poor prognosis after cardiac arrest in the Target Temperature Management trial. Resuscitation 131:24 28. https://doi.org/10.1016/j.resuscitation.2018.07.024

18. Sivaraju A, Gilmore EJ, Wira CR, Stevens A, Rampal N, Moeller JJ, Greer DM, Hirsch LJ, Gaspard N (2015) Prognostication of postcardiac arrest coma: early clinical and electroencephalographic predictors of outcome. Intensive Care Med 41(7):1264-1272. https:// doi.org/10.1007/s00134-015-3834-x

19. Young GB (2000) The EEG in coma. J Clin Neurophysiol 17(5):473-485

20. Steinlein O, Anokhin A, Yping M, Schalt E, Vogel F (1992) Localization of a gene for the human low-voltage EEG on 20q and genetic heterogeneity. Genomics 12(1):69-73. https://doi.org/10.1016/08887543(92)90408-k

21. Tjepkema-Cloostermans MC, Hindriks R, Hofmeijer J, van Putten MJ (2014) Generalized periodic discharges after acute cerebral ischemia: reflection of selective synaptic failure? Clin Neurophysiol 125(2):255-262. https://doi.org/10.1016/j.clinph.2013.08.005

22. van Putten MJ, Hofmeijer J (2015) Generalized periodic discharges: pathophysiology and clinical considerations. Epilepsy Behav 49:228-233. https://doi.org/10.1016/j.yebeh.2015.04.007

23. Renzel R, Baumann CR, Mothersill I, Poryazova R (2017) Persistent generalized periodic discharges: a specific marker of fatal outcome in cerebral hypoxia. Clin Neurophysiol 128(1):147-152. https://doi. org/10.1016/j.clinph.2016.10.091

24. Benghanem S, Paul M, Charpentier J, Rouhani S, Ben Hadj Salem O, Guillemet L, Legriel S, Bougouin W, Pene F, Chiche JD, Mira JP, Dumas F, Cariou A (2019) Value of EEG reactivity for prediction of neurologic outcome after cardiac arrest: Insights from the Parisian registry. Resuscitation 142:168-174. https://doi.org/10.1016/j.resus citation.2019.06.009

25. Hofmeijer J, Tjepkema-Cloostermans MC, van Putten MJ (2014) Burst-suppression with identical bursts: a distinct EEG pattern with poor outcome in postanoxic coma. Clin Neurophysiol 125(5):947954. https://doi.org/10.1016/j.clinph.2013.10.017

26. Vorderwulbecke BJ, Lichtner G, von Dincklage F, Holtkamp M (2018) Acute antiepileptic drug use in intensive care units. J Neurol 265(12):2841-2850. https://doi.org/10.1007/s00415-018-9069-3 
27. Schmidt D (1982) The influence of antiepileptic drugs on the electroencephalogram: a review of controlled clinical studies. Electroencephalogr Clin Neurophysiol Suppl 36:453-466

28. Geocadin RG, Peberdy MA, Lazar RM (2012) Poor survival after cardiac arrest resuscitation: a self-fulfilling prophecy or biologic destiny?*. Crit Care Med 40(3):979-980. https://doi.org/10.1097/ CCM.0b013e3182410146 